\title{
Research on Optimization Design of Suzhou Sandalwood Fans Based on Level of Analysis of Female Aesthetic Images
}

\author{
Lili Zhang $^{\dagger 1, a}$, Yue Qian ${ }^{\star 2, ~ b}$, Ming Xia ${ }^{1, c}$ \\ ${ }^{1}$ Visual Communication Design JiangSu University ZhenJiang, JiangSu, China
}

\begin{abstract}
This study constructs the hierarchical theoretical model of image design to realize more accurate aesthetic feature identification for females. The study mainly takes the scale, material and hollowed-out technique of Suzhou Sandalwood Fans as the carrier for the perception of products' aesthetic image, then retrieves words of female perceptual image with factor analysis in SPSS and other computer-aided information analysis methods, and evaluates the weights of different aesthetic image factors to screen out case models with higher adaptability, thus improving the market acceptance of subsequent design schemes. Based on relevant Suzhou cultural elements, it carries out the detailed optimization design practice to verify the feasibility of this method.
\end{abstract}

\section{Introduction}

Due to the impact of the consumer socialization and the change of women's social roles, contemporary females' aesthetics presents the characteristics of diversification and commercialization. Under such a historical background, it is required to be fully prospective and creative in the design process of female products. It is not only necessary to explore the value of the products themselves, but also to analyze the spiritual needs of customer groups and thus realize the combination of individuation and emotionalization. Suzhou Sandalwood Fan, as one of the four most famous fans in China, is a typical female product, and is mostly folding fans, which often adopts painting, garland, pyrographic and other decorative techniques presenting high artistry. Now as it is gradually fading out of people's sight, it faces difficulties in its development and inheritance. Therefore, strengthening user requirements analysis will incorporate requirements of modern female aesthetic images into the whole process of designs of Sandalwood Fans, so that the consumer can obtain the expected experiential value, thus helping sandalwood fans regain the public attention and produce greater market efficiency.

\section{Detailed Explanation Of "Image"}

In Rudolf Arnheim's aesthetic psychology theory, the word "image" is regarded as "the perceptual form abstracted from visual perception on the basis of observing objects' images, but "not a faithful copy of an object" [1]. "Female aesthetic image" means that the designer processes the product form from the perspective of women's psychological consciousness and aesthetic taste. The purpose of these aesthetic activities is to fully mobilize the various senses of the viewers and arouse their emotional resonance, thus improving the identification of the product.

Throughout the 100-year history of development of sandalwood fans, it is not difficult to find that Suzhou Sandalwood Fan manufacturers and workshops have been focusing on the modeling of fans to embody female aesthetic images. For example, on the basis of the original styles named after scenery like "Xiling" and "Jade Belt", they innovate new styles such as "Double Hollows" and "Hollow". Later, pyrographic techniques have been applied to fan decoration, supplemented by other decorative techniques such as garland and carving. The sandalwood fan with various styles and vivid patterns is popular among females.

\section{Creation Of Female Aesthetic Images In The Design Of Suzhou Sandalwood} Fan

Today's research on neuro-aesthetics holds that emotion as well as bottom-up and top-down neural processing determine the nature and degree of our aesthetics, which is an experience activity that touches emotion through sensory perception ${ }^{[2]}$. The generation of aesthetic images is the process of aesthetic activities, so the process of its creation can be divided into two major factors and three levels. One is "object", which refers to the external forms that can be perceived by the sense organs such as eyes and ears, including materials, colors, styles, etc., which can be defined as sensory image factors. The other is "meaning", which refers to the connotation and artistic conception, including culture, semantics and psychological experience,

\footnotetext{
a79summer@163.com

b*2015073975@qq.com

c1005157438@qq.com
} 
etc., which can be defined as emotional image factors. The complete design of images is constructed step by step among levels, as shown in Fig. 1, and embodies the unique mode of aesthetic thinking and creativity of the subject. ${ }^{[3]}$

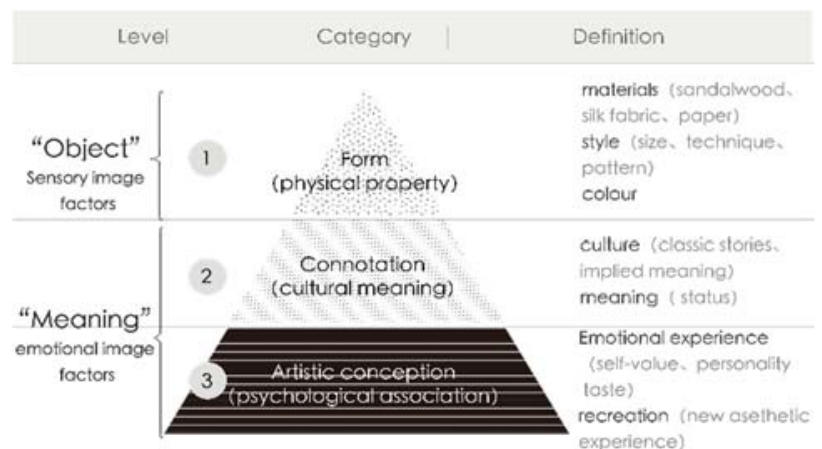

Figure 1. Hierarchical model of image construction of sandalwood fans

\subsection{Sensory image Factors}

\subsubsection{Unique fragrance}

Whether it is traditional or modern perfume, women's pursuit of fragrance has never stopped. One of the biggest characteristics of sandalwood fans is their rich fragrance of wood. According to the Market Report of Sandalwood Fragrance, since $1750,46 \%$ of some 7,000 classic perfumes contained sandalwood fragrance ${ }^{[4]}$. It can improve the hierarchy and durability of the overall fragrance of perfume. Because sandalwood belongs to prominent fragrance and its fragrance spreads widely, after a period of storage, the wood is rich in intoxicating fragrance, and has the effects of tranquilizing mind and uplifting spirits. Therefore, there is no synthetic essence or perfume comparable to natural sandalwood which is very popular among female consumers.

\subsubsection{Decorative patterns}

"Flower" is an indispensable and important element in the design of female aesthetic images, with graceful sensitivity. The techniques of "painting flowers", "garland" and "pyrographic" used in Suzhou Sandalwood Fans clearly reflect the characteristics of beautiful flowers, various flowers and elegant flowers. First of all, "painting flowers" commonly used in early silk sandalwood fans, refers to fine brushwork painting on silk and paper, mainly themed with peony, poppy and other four-season flowers. For cost reasons, this process has now been replaced by machine printing. "Garland" is one of the most important techniques to make sandalwood fans by carving hollowedout patterns with steel wire saws. It is famous for its difficulty of "strange, dangerous and skillful" operations. With the continuous improvement of tools for "garland", the patterns also gradually shift from rough and simple to delicate and complicated styles. Lastly, "pyrographic" also known as pyrography, is another technique developed after the 1940 s to beautify sandalwood fans. ${ }^{[5]}$ By controlling the temperature and time of the heater, the main patterns such as flowers can produce the hierarchical beauty of colors of the ink. The decorative patterns produced by the above techniques are a good match with the characteristics of flowers females are fond of.

\subsubsection{Small size in shape and structure}

The most basic requirement of product design is to meet the needs of users. Therefore, the most obvious change in the development of sandalwood fans is the increasingly prominent female characteristics in shape and structure. Compared with male fans, there are mainly the following two changes. First, the number of fan ribs and the range increases. Generally speaking, there are 16 fan ribs in folding fans for males, which have a spreading angle of $140^{\circ}$, and are easy to use due to low resistance. Thus, they are very popular. However, there are about $20 \sim 30$ fan ribs in fragrant wood fans such as sandalwood fans, and the range of opening and closing fans has a large span. Next, the 26 Suzhou Sandalwood Fans for females collected are measured in the range of opening and closing fans. Since the 1980s and 1990s were the heyday of sandalwood fan production and there were many classic styles then, the collected samples were mostly from this period.. Then the image processing software is used to desaturate the samples, and here are the clear results: the range of opening and closing sandalwood fans is about $118 \sim 176^{\circ}$ with an average of $152^{\circ}$, which is shown in Fig. 2. It has a larger range and better fanning effect than the male fans. What's more, the size is reduced and it is easy to carry. The folding fan is generally about $30-34 \mathrm{~cm}$ long, while the sandalwood fan is $20-30 \mathrm{~cm}$ long, which is shown in Fig. 3. Such change makes the sandalwood fan small, beautiful, easy to stretch, convenient to hold and easy to be put into bags. Secondly, the sandalwood has a long cycle of growing into useful timber. "The utilization rate of wood is less than $20 \%$. Per ton of sandalwood can only be made into 20 fans of 20 centimeters." [6] Small size in shape and structure can also help to save materials.

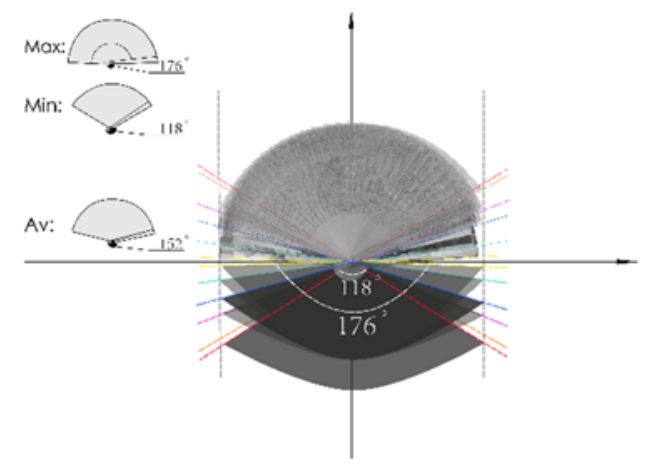

Figure 2. Schematic diagram of range of opening and closing sandalwood fans 


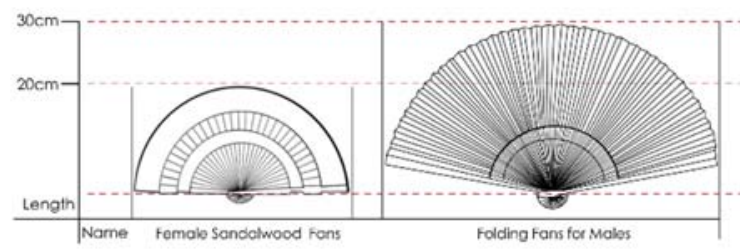

Figure 3. Comparison diagram of size of female sandalwood fans and folding fans for males

\subsection{Emotional image Factors}

\subsubsection{Expressing individual fashion}

"Consumer culture and fashion are important areas for females to reflect their personalization." ${ }^{[7]}$. Fashion magazines, advertisements and other traditional media have also produced an effect on the construction of sandalwood fan images. According to the two important periods in which females used folding fans in New Year pictures, calendars and magazine covers: Qing Dynasty (1636-1912) and Republic of China (1920-1935), these fragmented individual images are integrated into the mood board of collective images with similarities, which is shown in Fig. 4. From the figure, it can be seen that in Qing Dynasty, folding fans used by females were similar in appearance to male fans and were often used as opera props. By the time of the Republic of China, small folding fans, including sandalwood fans, had been frequently used by females living in the south of the Yangtze River, which was similar to what Suzhou writer Zhou Shoujuan described as "females love it very much and almost everyone has one." ${ }^{\text {[8] }}$ Usually this kind of fan is made of silk and painted with exquisite patterns, which can be used to go with fashionable dress and present the personality and character of users. These all reflect the functions of sandalwood fans which extend from practical function to decorative function.
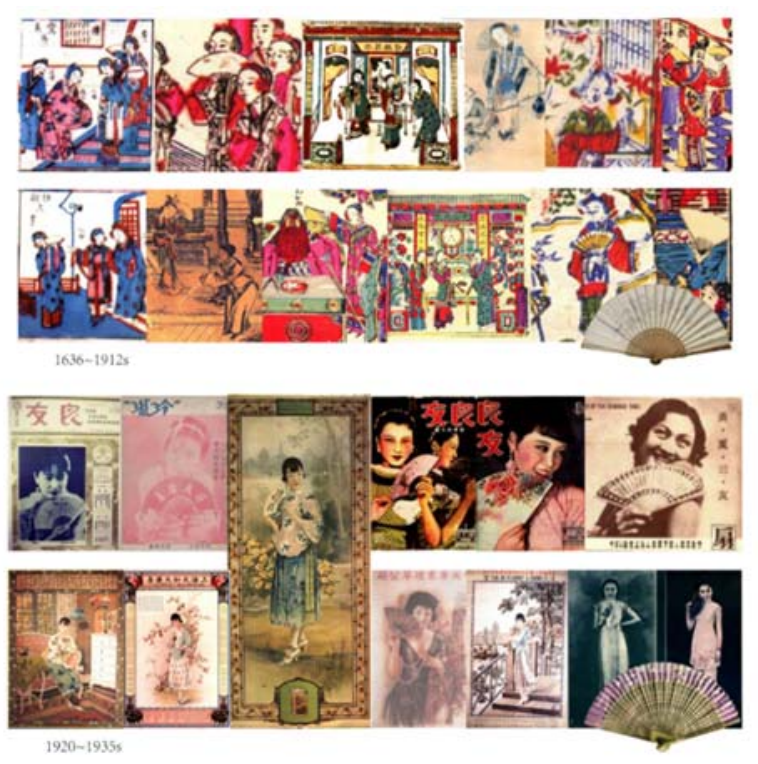

Figure 4.Mood boards of collective images of sandalwood fans in the two periods $1636 \sim 1912$ and 1920 1935

\subsubsection{Reflecting etiquette and taste}

In Europe in the sixteenth-century, delicate and exquisite oriental fans were very popular. The fan ribs were mostly made of high-end sandalwood or carved in ivory, with the highlight of openwork carving patterns. Different from the concept of elegant decoration and pursuit of natural wood texture in Suzhou sandalwood fans, such fans are often gorgeous and ornately decorated, and are the product of the integration of Chinese and Western cultures, extremely popular among the ladies of the court. So they often take it with them when attending the dance party or banquet, believing that it can reflect elegant social etiquette. At the same time, waving the fan is full of emotional appeal and can be used to show their attitude: refusal or acceptance ${ }^{[9]}$. But in China, females also use sandalwood fans to cover their faces to hide their inner shyness and set off their reserved temperament. These different ways of using fans are all to express the unique aesthetic taste and subtle emotional characteristics of females.

\section{Optimization Design Of Image That Embody Modern Female Aesthetics}

Although the creators in the past tried their best to think about how to express female aesthetic taste in sandalwood fans, they would eventually be constrained by the artist's personal perspective and leave a social mark on the products. Consequently, continuously injecting new aesthetic experience can prevent sandalwood fans from being washed out by the times. Next, this paper explores the method of image positioning for the external contour which is the typical style feature of sandalwood fans, and optimizes designs of garland patterns of the fan, so as to provide reference for style designs and present universal and shared value.

\subsection{Contour and style analysis of Sandalwood Fans}

\subsubsection{Positioning of words of perceptual images for the contour of Sandalwood fans}

First of all, the objects should be determined, namely the external contour line of sandalwood fans. In the design of industrial products, the style of an object is composed of a combination of various features which can be divided into feature points, feature lines and feature surfaces. For example, the feature lines of automobiles are waist lines, broadside lines, etc. Similarly, sandalwood fans also have their own feature lines, of which the most vivid and visual focus is the external contour line, which refers to the curved line at the edge of the fan. It is usually designed into various dynamic styles.

Then, 20 groups of adjectives of perceptual images are collected and selected from three image targets and then are combined into word pairs to represent women's expectation of styling with contour feature lines of the fan, as shown in Table 1. Then the target group is 50 females aged between 25 and 40 . At this stage, people are economically independent and pay more attention to the 
quality and connotation of products. Then the questionnaires are designed based on semantic differential method, letting the target group to evaluate the abovementioned word pairs.

Table1. Words Pairs Of Perceptual Images

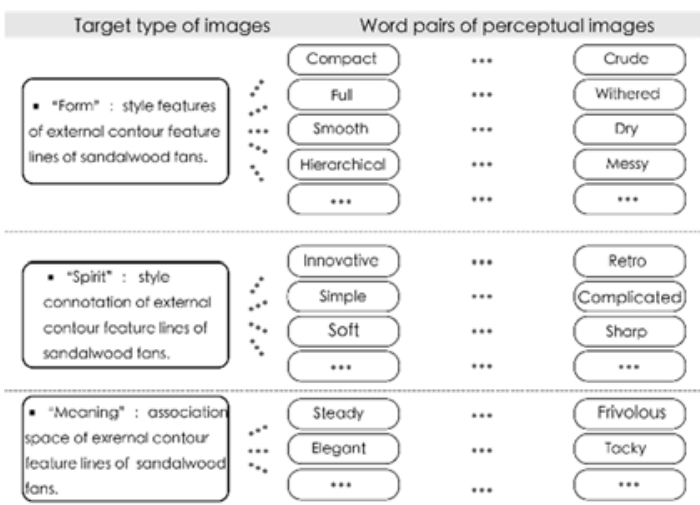

The cognitive space composed of evaluation words is too complex and the dimension needs to be reduced through factor analysis. ${ }^{[10]}$ Factor analysis is the study of the internal structure of matrix by combining the correlation coefficients of variables and its main process is to find out a few random variables that can control all variables to describe the correlation between multiple variables, and these random variables which are difficult to observe are often called factors. ${ }^{[11]}$.

Through factor analysis of SPSS software, 10 word pairs of perceptual images are screened out. The results can be seen in Table 2. Several indexes can be divided into three categories according to high load. It is concluded that the image words of the external contour of sandalwood fans are mainly explained by three factors, and then cluster analysis is carried out on this basis. Finally, three pairs of image words conforming to the description of perceptual images are extracted, namely, "smooth-dry", "eleganttacky" and "innovative-retro".

Table2. Image Factor Analysis Of Female Users' Expectation Based On Questionnaires

\begin{tabular}{lllll}
\hline \multirow{2}{*}{ Factors } & Vocabulary & \multicolumn{3}{c}{ Load coefficient } \\
& Smooth & 0.593 & -0.232 & -0.186 \\
\multirow{3}{*}{ Factor 1} & Soft & -0.753 & -0.160 & -0.072 \\
& Innovative & 0.476 & -0.2334 & -0.210 \\
& Elaborate & 0.579 & 0.136 & -0.119 \\
\hline \multirow{2}{*}{ Factor 2 } & Compact & 0.065 & 0.705 & -0.127 \\
& Elegant & 0.091 & 0.832 & 0.100 \\
& Full & -0.162 & 0.747 & -0.271 \\
\hline \multirow{6}{*}{ Factor 3 } & Steady & 0.095 & 0.188 & 0.879 \\
& Rhythmical & -0.083 & -0.129 & 0.722 \\
& Hierarchical & 0.067 & 0.175 & 0.799 \\
\hline
\end{tabular}

\subsubsection{Typical cases screened out of various styles}

In order to select styles of the sandalwood fan with high adaptability as typical cases for the reference of designers, it is necessary to evaluate and score images of the contour line of the collected sandalwood fan samples through the above-mentioned image adjectives of users' expectation (smooth, elegant and innovative). First of all, samples of Suzhou sandalwood fans selected from the market should satisfy the following three conditions: first, they are wellsold, which proves they are popular among the public; second, the edge shape of the fan cannot be the same and has distinctive features; third, the basic dimensions are similar. Therefore, 12 groups of cases satisfying the above-mentioned conditions are obtained, and then the contour feature line of the edge of the fan is obtained by drawing Bezier Curve, which helps to eliminate other interference items during investigation. The Bezier Curve is used here because it is a free curve, and the shape of the line can be changed with the variation of the position and weight of the control points. ${ }^{[12]}$ It can clearly display the changes of lines. Finally, according to the results of the questionnaire, 5 sandalwood fans with higher scores were selected, which is shown in Fig. 5. It can be seen that the overall contour curve tends to be slightly dynamic without obvious sharp bulges, and the style of a single bamboo strip is round and full. Such characteristics conform to modern female aesthetics.

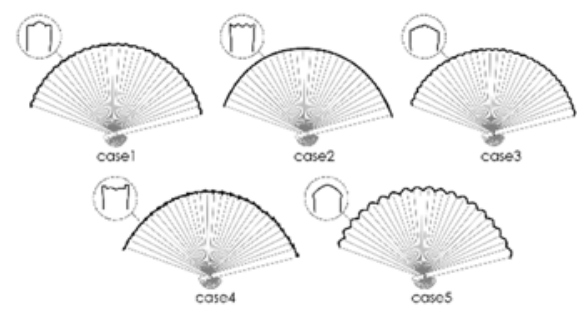

Figure 5. Case groups of contour lines of sandalwood fans with high image conformity

\subsection{Design of decorative patterns on Sandalwood Fans}

The hierarchy diagram of image designs shows that the ultimate goal of the design is to be user-centered, not only satisfying the functional value, but also satisfying the emotional experience of users. First of all, in terms of function, the sandalwood fan is defined as "Wedding Fans", which makes it a beautiful wedding or festival accessory. Considering the source of the pattern, it fully demonstrates Suzhou's regional culture. When we relate it to Suzhou's local products, we have in mind garden architecture, whose pane pattern of its flower window is in line with the characteristics of garland of sandalwood fans to some extent. Therefore, in the Humble Administrator's Garden, the representative building of the gardens, two kinds of flower windows that conform to the positioning are selected as the basic patterns: pomegranate-shaped flower windows that symbolize "happiness lies in having many children" and Xi-shaped flower windows that symbolize "here comes the happiness". The patterns are extracted and evolved with the concepts of "good luck" and "simplicity", as shown in Fig. 6 and Fig. 7. 


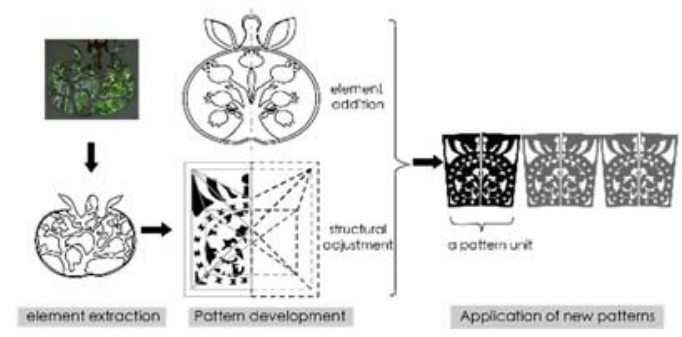

Figure 6. Partial pattern designs of "Pomegranate-shaped" edge of fans

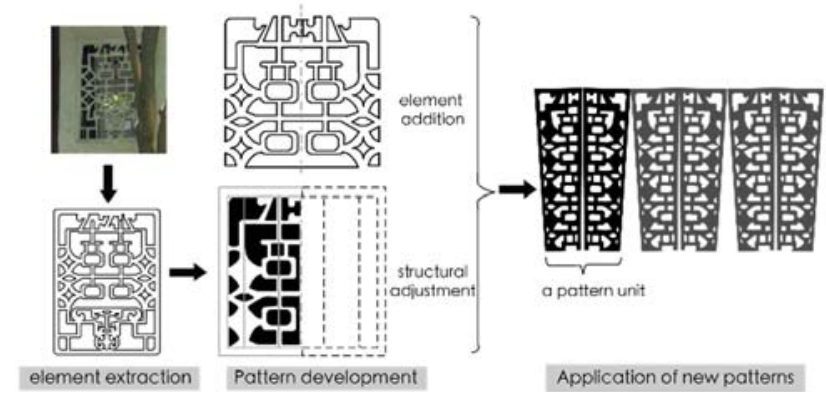

Figure 7. Partial pattern design of "Double Xi" fans

"The connotation of a pattern is the design and artistic conception realized by artistic means before the manufacture of objects or the arrangement of the environment." [13] When designing sandalwood fan patterns, one cannot deviate from its characteristics in shape and structure. Bamboo strips of the fan are long and narrow inverted trapezoids with large topside area and small bottom area, so at the top of the fan, the edge part uses a slightly complicated pomegranate-shaped quadratic sequence pattern, that is, a pattern unit extends to both the left and right and looks neat and unified. In the middle part, a simpler quadratic sequence pattern of "double Xi" characters is applied, but the part of $\mathrm{Xi}$ character in the pattern separately presents the design method of quadrilateral sequence patterns, that is, a pattern unit extends up and down, and to the left and right, looking fine but orderly. In a word, the overall design follows the rule of garland pattern of sandalwood fans: two-stage layout is adopted; the pattern is formed by connecting small pieces of surface; the holes are fewer when it is closer to the bottom, and the hollowing is stopped at a distance of one third from the bottom. The "Wedding Fans" pattern finally designed is variable in density, which not only retains the traditional charm but also adds new ideas in form, thus achieving the purpose of optimization design.

\section{Conclusion}

Suzhou sandalwood fan is not only an important tool for women to harness the wind and enjoy the cool air, but also one of the important carriers to spread the South-China culture. It has extremely high technological value, cultural value and economic value. In order to make sandalwood fans more attuned to modern people's aesthetic taste, under the mode of quantitative analysis, this paper discusses the corresponding relationship between the psychological perceptual image of females, the main consumers of sandalwood fans, and the feature elements of sandalwood fans. With clear functional objectives, transboundary thinking is used to extract relevant elements of garden architecture, and the design of visual focus of sandalwood fan's styles, namely garland pattern is optimized, which can better satisfy the aesthetic needs of female consumers and enrich the style designs of sandalwood fans.

\section{Acknowledgement}

Philosophy and social science of Jiangsu Province "Research on cultural Derivatives Innovation of Jiangsu section of the Grand Canal Based on image differentiation strategy" (Project No: 2020SJA2069).

\section{References}

1. Rudolf Arnheim. Visual Thinking-Aesthetic Intuition Psychology[M]. Teng Shouyao. Trans. Chengdu: Sichuan People's Publishing House, 1998:136.

2. Ann Marie Barry. Perceptual Aesthetics: Transcendent Emotion, Neurological Image[J]. Visual Communication Quarterly,2006,13(3):134151.

3. Zhu Zhirong. On the Creation and Construction of Aesthetic Image. Art Monthly[J].2014,5(46): 110.

4. Market report of sandalwood fragrance. Domestic and foreign fragrance information[J].2015(08):8.

5. Compiled by Suzhou Sandalwood Fan Factory. Fanmaking technology[M]. Nanjing: Jiangsu Science and Technology Press, 1989: 19.

6. Song Lan.A survey of Jiangsu Suzhou Ruyi Sandalwood Fan Co., Ltd.[J]. Popular Literature and Art, 2010,25(5):100-105.

7. C Delhaye.The Development of Consumption Culture and the Individualization of Female Identity Fashion discourse in the Netherlands 1880-1920. [J].Journal of Consumer Culture, 2006:87.

8. Zhou Shoujuan. Flowers, flowers and grass[M]. Shanghai: Shanghai Culture Publishing House, 1956: 68.

9. PIZZO T. The Fan[J]. Tampa Historical Society, 1991:17.

10. Su Jianning,Li Heqi,Li Fenqiang.Study on Perceptual Image Positioning in Product Design[J].Journal of Lanzhou University of Technology, 2004, 30(2):4043.

11. Wei Yanhua,Wang Bingshen,Tian Yuzhu.The comparative study of principal component analysis and factor analysis[J].Journal of Tianshui Normal University, 2009,29(2):13-15.

12. Fu Pengfei.The application of Bezier curve in automobile design[J].Enterprise Technology Development, 2012,31(26):46-47.

13. Zhang Daoyi. Collected Works of Zhang Daoyi (Part 1) [M]. Hefei: Anhui Education Press, 1999:267. 